
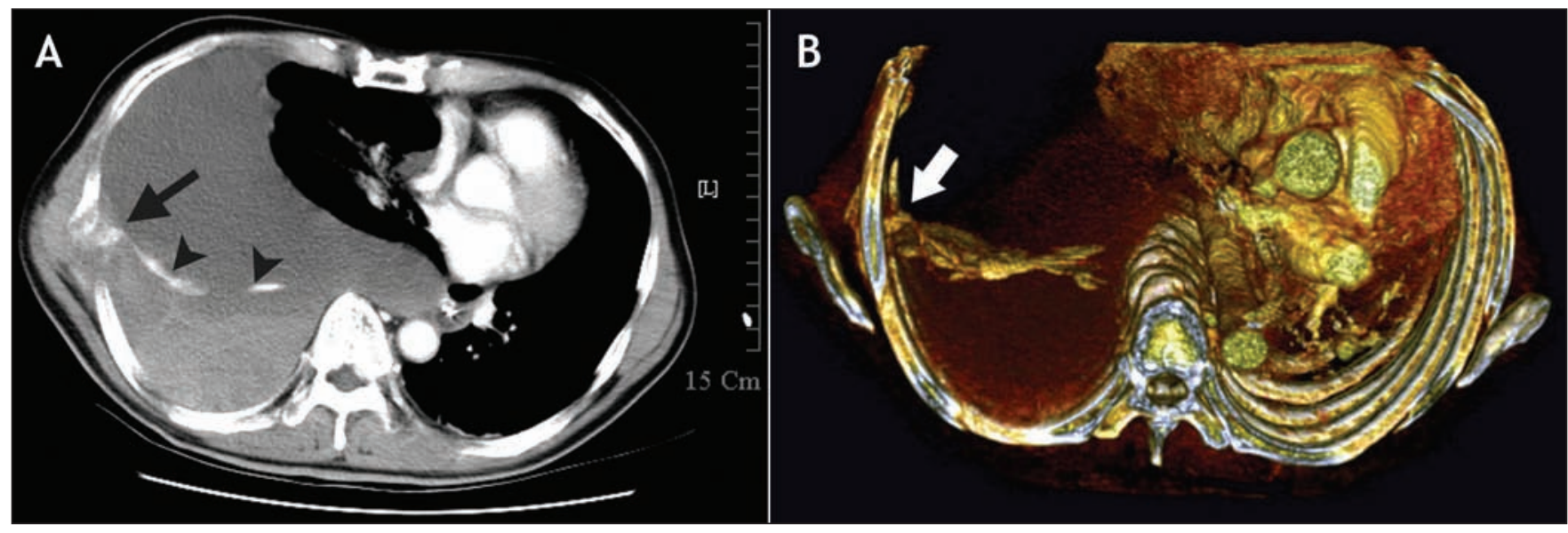

Figure 1: A: Chest computed tomography scan with contrast showing extravasation of the contrast medium (arrow) from a rib tumour and a sheet (arrowheads) separating different density pleural fluids. B: Coloured 3-dimensional reconstruction of the computed tomograpy scan showing the bleeding site (arrow).

\title{
Spontaneous hemothorax caused by metastasis of a rib tumour
}

$\Delta$ 62-year-old man with a history of hepatocellular carcinoma presented to the emergency department with sudden onset of chest pain on his right side, dyspnea and syncope. A chest computed tomography scan with contrast showed active extravasation of the contrast medium from a rib tumour, forming a sheet separating pleural fluids of 2 different densities (Figure IA). A coloured 3-dimensional reconstruction of the computed tomography scan showed the bleeding site (Figure $\mathrm{IB}$ ). Bone-window and precontrast views confirmed that the higher-density material was contrast agent (Appendix I, available online at www.cmaj.ca/cgi/content/full/178/6/679/DCI). Thoracocentesis yielded a bloody effusion, which confirmed the diagnosis of a hemothorax. The most common cause of hemothorax is trauma. Spontaneous hemothorax can be caused by a primary or $m$ metastatic neoplasm, aortic dissection, coagulation disorders, endometriosis and pulmonary infarction. Hemoperitoneum caused by a ruptured primary tumour is a wellknown presentation of hepatocellular carcinoma; however, hemothorax caused by the rupture of a metastatic hepatocellular carcinoma is very rare. Treatment options for spontaneous hemothorax because of metastasis of a rib tumour include tube thoracotomy, surgical hemostasis, transcatheter arterial embolization and percutaneous ethanol injection. However, as in our case, most patients die as a result of uncontrollable hemorrhage.
$C M A J$ invites contributions to Interesting images, a new column with a very brief but clear description of the case, the images and the main teaching point. Submit manuscripts online at http://mc.manuscriptcentral.com/cmaj.
Che-Kim Tan MD

Department of Intensive Care Medicine

Kuo-Chin Wu MD

Department of Emergency Medicine Reng-Hong Wu MD

Department of Radiology

Yu-Hui Lui MD

Department of Emergency Medicine Chi-Mei Medical Center

Yungkang, Taiwan 